\title{
A Review of Feature Selection and Sentiment Analysis Technique in Issues of Propaganda
}

\author{
Siti Rohaidah Ahmad ${ }^{1}$, Muhammad Zakwan Muhammad Rodzi ${ }^{2}$, Nurlaila Syafira Shapiei ${ }^{3}$ \\ Nurhafizah Moziyana Mohd Yusop ${ }^{4}$, Suhaila Ismail ${ }^{5}$ \\ Department of Science Computer, Faculty of Defence Science and Technology \\ Universiti Pertahanan Nasional Malaysia, Sungai Besi, Kuala Lumpur, Malaysia
}

\begin{abstract}
Propaganda is a form of communication that is used in influencing communities, or people in general, to push forward an agenda for a certain goal. Nowadays, there are different means used in distributing propaganda including postings on social media, illustrations, cartoons and animations, articles, TV and radio shows. This paper is focused on election propaganda. Candidates in elections would use propaganda as a form of communication to channel and deliver messages through social media. Sentiment analysis (SA) is then used in identifying the positive and negative elements within the propaganda itself, through analysing the related documents, social media, articles or forums. This paper presents the various techniques used by previous researchers in issues of propaganda using $\mathrm{SA}$, which include feature selection to remove irrelevant features and sentiment methods to identify sentiment in documents or others. Feature selection is a dominant side in sentiment analysis due to content of textual has a high measurement classification that can jeopardize SA classification interpretation. This paper also explores several SA techniques to identify sentiments in issues of propaganda. This study has also attempted to identify the use of swarm algorithms as a suitable feature selection method in SA for propaganda issues.
\end{abstract}

Keywords-Sentiment analysis; feature selection; swarm algorithm; propaganda

\section{INTRODUCTION}

Social media networks, such as Facebook, Twitter, and Instagram can be accessed online to facilitate a user's interaction with another user, such as sharing news, reading, discussing important events, having political discussions, and sharing thoughts. Smartphones have also helped to spread the use of social media to unlimited duration and location. This technology has facilitated in spreading information quickly and accurately to users. Propaganda would normally consist of accurate or semi accurate information, which is used to influence people to spread the various agendas to achieve a specific goal.

According to [1], propaganda is a special kind of social communication that involves three conditions, namely, the nature of its target society, communication experience, and the propagandist. In [2], propaganda refers to any communication used to assist or support the objectives of the government or the opposition in persuading, and influencing the emotion, behaviour, and actions of an individual, or any groups for the benefit of the propagandist, either directly or indirectly. Propaganda is used to influence the minds of millions of people as they go about their daily lives using various modes of communication (Lasswell, 1927). Although propaganda is often linked to the dissemination of negative messages [3] that are aimed at gaining or turning the support for the opposing party towards the propagating party, propaganda can also be used to spread positive messages [4], such as championing women's rights, racial equality, community health, encouraging people to vote, and so on. In conclusion, propaganda can be used to convey information that contains either positive or negative elements. Sentiment analysis (SA) is used to extract positive, negative, or neutral sentiments from text data. The results of this analysis can help various organisations in various fields, such as politics, business, productions, and marketing to make decisions. Thus, sentiment analysis can be utilised by certain parties to analyse and determine the status of a message, whether it contains positive or negative propaganda issues. This study aims to extensively analyse the effectiveness of SA for issues of propaganda, especially in political focus during elections.

The paper structure is as follows; Section 2 will display and expose the generic information about propaganda. Section 3 discusses about SA and how it can be linked to propaganda. In Section 4, feature selection and SA techniques are discussed in relation to issues of propaganda. Section 5 explains about a survey of swarm algorithms as a feature selection technique in SA. Finally, the last section will focus on the conclusions and possible directions for future research development plan.

\section{PROPAGANDA}

Propaganda can be defined as an act of disseminating rumours, anecdotes, stories and gossips, whether it is based on a true or false allegation, with the sole purpose of persuading and influencing the public. The normal practice is that propaganda is used to influence people's mind in a misleading way by providing fabricated facts. In literature writing, propaganda is often referred to as a technique in controlling and manipulating the society's beliefs. History shows that propaganda is used in shaping the public's perception and discernment in order to achieve the influencer's political or economic goal [5]. During the First World War at around the early twentieth century, the word 'propaganda' began to undergo changes in its role and task, from religious indoctrination to political aspect or views, which reflect the shift in societal power from Church to State [6]. It was during this period that propaganda emerged as being political and partisan in nature, with its attempt to coerce or persuade a 
mass audience to conform to a particular opinion or point of view. Based on the above definitions, propaganda can only be created through deliberate planning involving current sentiments from propagandists. Several questions have been raised regarding why propaganda exists. In [7], the objective of a propaganda may be to impress people to accept beliefs and attitudes pertaining to those of the propagandist or to bemuse certain patterns of behaviour, such as contribution of money, joining groups or blast-off demonstration for a cause. They also explained another objective of propaganda, which is to protect and maintain the authority of the institution or organisation it represents to ensure the legitimacy of its campaigns and events. Thus, it can be concluded that propaganda is created only to achieve the propagandist's dogma. Hence, propaganda activities are important to shape people's mind set in accepting the propagandist's ideology.

\section{SENTIMENT ANALYSIS}

Sentiment analysis (SA) involves analysing texts that contain opinions and emotions. Any opinion or emotion expressed in the form of a text would contain a negative, positive, or neutral element. The information or opinion exchange and propaganda procreative often used in the internet [8]. According to [8], although different types of text classifications are available, SA was chosen for two main reasons. First, web pages are rich in emotion-related opinions and content. Second, text analysis relates to the use of propaganda on the web because text content contains opinions or directives that could influence public perception in decision making. [8] Also argued that SA is capable of detecting the presence of propaganda, and anger or hatred embodied in community channels. In addition, [8] concluded that SA can help produce an effective analysis and better understanding of the use of the web by extremist groups for disseminating information and propaganda. In the field of politics, [10] [13] used SA to analyse sentiments contained in speeches of candidates for elections, issues, and prediction of election results. Propaganda is also used by the military, media, politicians, advertisers, governments, businesses, during election, marketing and by other mediums. This study will examine how previous studies utilised SA to analyse the propaganda contained in the speeches of candidates during elections.

\section{A REVIEW OF FEATURE SELECTION AND SENTIMENT ANALYSIS TECHNIQUES}

The purpose of this study is to identify two main points in selected literatures discussed in this paper. The first step was to identify the feature selection or feature extraction techniques used to select important and useful features. The second step was recognising the methods used to identify the sentiments in the speech of an electoral candidate. According to [13], a feature is an aspect or attribute data [14] that users commented on. For example, a Panasonic electrical appliance, such as a radio is the product feature. Meanwhile, sentiment includes the words used to describe negative or positive sentiments, such as bad, good, beautiful, amazing, and so forth. In [13], feature can be categorized in two groups. First, in the form of unigram, bi-gram or tri-gram from a list of word in a document. Second using a part of speech tagging to identify each word in the sentence whether the word is a noun, adjective, adverb, verb, determiner or preposition.

Ramteke et al. [9] suggested two level or stage of framework in order to create a labelled training dataset. In stage one; they used hashtag clustering to get several tweets that consists similar hashtag. For example, the official hashtag for the American presidential candidate, Donald Trump, was \#MakeAmericaGreatAgain. Therefore, anyone who tweeted using this official hashtag was considered to support Trump and labelled as a positive tweet. In stage two, they used Vader to analyse sentences as the input and to produce a percentage value for three categories, namely, positive, negative, and neutral and compound for the overall polarity of the sentence. In this experiment, they used Naïve Bayes and Support vector Machine (SVM) as the supervised learning algorithm in order to adjudicate the contrariety of tweets and predict election outcomes. They collected twitter data for two U.S. Presidential candidates, which were Donald Trump and Hillary Clinton, from 16 - 17 March 2016. They used TF-IDF (term frequency-inverse document frequency) to recognise terms that were applicable towards specific sentiments. Meanwhile, [10] did not use feature selection techniques or feature extraction to choose useful features. They used Syuzhet [15] methods to identify sentiments in the speeches presented by election candidates. A machine-based method and a structural topic-modelling [16] were used to analyse themes in candidate speeches. The results of the 50 main terms used in speeches were identified. In the final step, the word2Vector was used to sketch the candidates' words from specific thematic terms. The dataset collected from Clinton's speech included 89 scripts; with a total of words are 286,899. As for Trump's speech, 74 scripts were collected, with a total of 276,212 words. This dataset was collected from 1 April 2015 to 9 November 2016 for Clinton, whereas the dataset for Trump was collected starting from 16 June 2015 to 9 Nov 2016. Experimental results showed that a combination of machine-based methods of stm, syuzhet, and word2vec was very effective and quick to identify sentiments and themes in speeches. According to the authors, the combination of stm and word2vec had helped them understand the deeper perspective of the candidates' attitudes, views, and perspectives on the issues being reviewed. These researchers have also assumed that qualitative analysis is required to ensure that important information in the speech of a candidate is not lost.

Researchers [11] were able to classify texts related to the presidential and gubernatorial candidates for the Sao Paulo 2014 elections in Brazil. A dataset of 131 online news articles related to the 2014 elections was collected from Veja, Estadao, Folha, GI, and Carta Capital. They aimed to classify every paragraph in each article into either a positive, negative, or neutral group. Three learning algorithms, namely, Naïve Bayes, Support Vector Machine, and MaxEnt were used for sentiment classification. They conducted two experiments; first, they used the combination of unigram and bigram as a feature extraction technique to evaluate the best paragraph representative. Second, they also used feature selection method such as Chi-square, Categorical Proportion Difference, and Categorical Probability Proportion Difference 
(CCPD) for selecting features. This second experiment was conducted to assess the effect of feature selection on learning algorithms. Experimental results showed that the MaxEnt learning algorithms, which use unigrams and bigram combinations, and was selected by CCPD that acts as feature selection technique was the most effective method for classifying text.

Researchers [12] used the Storm-based Real-time Analytic Service (SRTAS) designed to excerpt political-related tweet data from public tweets. This study had also analysed propaganda and sentiment rates using SRTAS and Kafka, namely, the Big Data System. Data tweets went through data processing, and each tweet was classified using the SVM module. Tokenizer was used to identify meaningful terms, and the stemming process was conducted to reduce the token that was relevant to the individual token. The process of classifying sentiments is often based on word subjectivity. Each word need to compare with predefined word-net libraries (AFINN-111) by researchers. However, their study did not specify the measurements used to measure the effectiveness of SRTAS. They also did not use feature selection techniques to choose relevant features from public data tweets.

Research in [17] conducted sentiment analysis on 42,235 twitter archive data in Hindi language using Dictionary-based, Naïve Bayes, and SVM algorithms. They analysed the sentiments of twitter users towards five political parties during India's general state election in 2016. Through supervised and unsupervised approaches, they built a classifier that could classify the collected data into positive, neutral, and negative groups. The limitation of their research was that they failed to consider the emoticons used in a message, which are relevant when defining the polarity of a tweet. Results of their analysis using SVM and Naive Bayes techniques showed more positive sentiments for the Bhartiya Janta Party (BJP), while the Dictionary-based approach showed more positive sentiments for the Indian National Congress. The SVM also read that BJP has a $78.4 \%$ chance to win the election based on the positive tweets. However, the final results showed BJP had only won 60 from 126 constituencies during the general election. The authors [18] also performed sentiment analysis on 343,645 twitter messages during the Austrian Presidential election in 2016. The dataset included tweets from the two presidential candidates, Van-Derbellen and Norbertghofer. The researchers used a combination of network science and sentiment analysis methods. They ran the SentiStrength algorithm during data preparation regarding on a lexicon of sentiment words, idioms, and emoticons in order to separate positive and negative data. Besides, they also occupied the NRC emotion-word lexicon through the tweets, and then stored all the identified emoticons in the tweets. Next, they conducted data analysis for the final version using sentiment analysis, network analysis, text mining techniques, and quantitative data analysis. Some limitations were observed when using SentiStrength and the NRC emotion-word lexicon. Thus, the limitations show some scores were inappropriate when assigned by these tools. In [19], the researchers used the lexicon and Naive Bayes Algorithm Learning Machine (NBALM) to totalize the political sentiments expressed in tweets for 100 days earlier from the election day. However, NBALM was only able to identify sentiments related to certain hashtags compared to lexicons of analysis. These researchers labelled the tweets manually and automatically. As a result, labelled tweets automatically had better precision than lexicon analysis. Therefore, the labelling of tweets automatically saved working hours, improved accuracy, and discarded any potential tendencies.

In 2017, the authors [20] used Twitter to determine sentiments in elections. The dataset was collected based on hashtags by two presidential candidates (Trumph and Hillary) starting from 24 April till 28 November 2016. Analysis of Variance (ANOVA) was accomplished using IBM SPSS to facilitate the validation of sentiment analysis results. This study was able to identify positive and negative sentiments that could be influenced by the debates, controversies, interviews, and other revelatory events. Nonetheless, they did encounter several limitations when contextualising the sentiments found in these tweets: limited number of characters for each tweet (only 140 characters are permissible); only a small number of tweets were used within the timeframe of their study; Twitter API was used to support composing or gaining the tweet (thus, difficulties in interpreting the exact meaning of a tweet); limited hashtag usage for both candidates; and no geographical mapping of the sentiments were provided.

\section{A SURVEY ON FEATURE SELECTION USING SWARM ALGORITHM IN SENTIMENT ANALYSIS}

Swarm Intelligence involves a simple assemblage of agents interacting locally and externally in their corresponding circumstances [21]. The individual agent is unintelligent, but the overall system of Swarm Intelligence commands the intelligent comportment with interactions between the agents and their environment. Swarm Intelligence algorithms are broadly used in optimisation problems where the calibre of result could be scaled. These algorithms will improve the quality of the solution by working in several iterations or repetitions and knowledge application of previous iterations on a selection of recent values. A comparison between various Swarm Intelligence techniques has shown that exactitude with much reduced feature sets can be achieved. In this comparison, three Swarm Intelligence techniques were used, namely, the Artificial Bee Colony (ABC), the Ant Colony Optimization (ACO), and the Particle Swarm Optimization (PSO). This comparison experiment used datasets from product reviews, internet movie database (IMDb), governmental decisions data, Twitter data, and restaurant reviews. The $\mathrm{ABC}$ and $\mathrm{PSO}$ algorithms, being puissant optimisation techniques, are widely used for working out hybrid optimisation challenges. These two methods have been used for optimising feature selection subsets, and for improving the accuracy of classification and clustering. The PSO algorithm, when amalgamated with sentiment classifiers (SVM and CRF), can enhance the classification accuracy by 4.25\%. Meanwhile, the ABC algorithm often applies SVM, Naïve Bayes, FURIA, and RIDOR as classifiers, with accuracy increments of $9.04 \%$ as concluded in this research, Swarm Intelligence algorithms have generated better results based on sensitivity, specificity, and accuracy. It could also improve a classifier's performance in each SA. 
Researchers in [22] reported that feature selection methods are necessary when selecting relevant feature vectors. One of the most consequential processing steps in classification is feature selection. This step is important for choosing useful features that would not affect the quality of the classification. They proposed the fitness proportionate selection-based binary particle swarm optimisation (F-BPSO) as a feature selection method in their study. To manufacture some changes in the experiment, these researchers used fitness sum rather than average of fitness in the fitness proportionate selection step. This modified approach is known as the fitness sum proportionate selection binary particle swarm optimisation (FS-BPSO). The FS-BPSO method did the final changes to make it more appropriate for the feature selection specialty, which is oriented towards sentiment classification. This further modified method is called the SCO-FS-BPSO, where SCO refers to "sentiment classification oriented". Experimental results showed that the SCO-FS-BPO can produce higher accuracy and obtain better quality features compared to the traditional Binary Particle Swarm Optimization (BPSO) and F-BPSO. This study used two benchmark datasets, namely, the Madelon and the Semeion Handwritten Digit (shortened to Semeion for simplicity) to test the effectiveness of the proposed method.

Humans around the world are spending multitudinous time using the internet, especially when surfing various social networks (Web 2.0), such as Facebook, Twitter, and Instagram. Hence, these social media which produce many opinions had influenced almost every single human in this world [23]. Thus, [23] have tried to understand this phenomena by using the revolutionary swarm intelligence algorithms, which might be the best and the most similar to the interactions between online users as members of a swarm. They used two datasets from Twitter and Reddit API. Twitter data format must include 'User_id', 'id_str', 'created_at', 'favourite_count', 'retweet_count', 'followers_count', and 'text'. Meanwhile, Reddit data consists of simple plain texts, with no limit to the length of posts and paragraph usage. In addition, Reddit only stored the data for all post posted by user which contains a string of text. Ten different subsets were divided from the dataset. Nine subsets were used for training and the final subset was used for testing. Two braces of heuristic arrays and pheromone continued to update their previous values and predictions during training. These arrays were called positive pheromones, negative pheromones, positive heuristics, and negative heuristics. The progression of the ant colony system for this experiment was trailed by these four arrays, which was also used to assist the ants knows how to structure the right conclusion. Other than that, all sentiments of posting were evaluated using natural language processing (NLP) techniques. The value for correct and incorrect predictions will increase if a post contains sentiments and the prediction matches of the sentiment. The results showed that the prediction accuracy for Twitter data was higher than for Reddit data. This research had also presented a graph of increasing positive heuristic and decreasing negative heuristic. Thus, it was concluded that one of the advantages of a swarm intelligence algorithm is its ability to perform faster and better than traditional algorithms for Sentiment Analysis, partly because it closely resembles human behaviour. Besides, it is a good algorithm in order to break down sentiments in social sites. Nonetheless, when sentiment changes rapidly and drastically like in group of chats, this algorithm does not perform well.

Feature selection is the main step in classification system that selects a subset from original features. In [24], four features selection were conducted in the comparative study for text categorization that include Information Gain (IG), CHIsquare, Genetic Algorithm (GA) and PSO in order to optimize classification result. The performance of this propose method have been tested using Reuters-21578 dataset. Besides that, in order to show effectiveness of this experiment, they used a simple classifier, K-Nearest Neighbour (KNN) that effects the performance of categorization. After analysing the precision and recall, the PSO-based algorithm showed a better reading accuracy value than other three of feature selections. The percentage of selected features achieve until $12 \%$ in micro-F1 and macro-F1 measures when tested with PSO-based algorithm. Hence, PSO-based algorithm is the best compare to GA, IG and CHI with reading in Macro-F1 is 78.8564 and Micro F-1, 89.5684 [24]. PSO is quickest in locating optimal solution and able to get the determination within 10 iterations. Several advantages that can be identified from this propose method such as PSO has the capability to assemble quickly where it has strong searching skills, able to identify the problems in the environment and can effectively search the slightest feature subset within the sentence [24]. The drawback which can be listed is all the values from population size, maximum number iterations and range of weight are not optimal values. This disadvantage can be discussed in further research.

The growth of social media contributes to big numbers of user generated content including opinions, client reviews and comments. So, it is one of the opportunity to evolve an intelligent system that spontaneously cluster and classify them into positive and negative [25]. In this experiment, two classifiers were used, SVM and the hybridization of two classifiers, SVM and PSO (SVM-PSO). The multiplication of TF and IDF will calculate the score of every sentence in source document which word based on adjective word excerpted from Parts of Speech Tags using movie review dataset [25]. The approaches of sentiment analysis in [25] for sentiment analysis involve a two-level method, firstly, recognize the parts of document in order to provide the positive or negative sentiments. Secondly, connecting these parts of document in ways that boost up the odds of document falling into one of these two types of categories. This research has categorized data into four groups, True Positive (TP), False Positive (FP), True Negative (TN) and False Negative $(\mathrm{FN})$. The result of SVM-PSO in precision, recall and accuracy test is better than SVM [25]. One of advantages can be concluded in this research is the hybridization of SVMPSO increases the accuracy reading in sentiment analysis. Besides, this research help in making decision towards client reviews, comments and opinions and can save more consume of time in analysis [25]. Despite, the drawback in this research is the small number of classifiers which has only two. In order to yield the best result, more classifiers should be involved in experiment. 
TABLE. I. A SUMmary of FEATURE SELECtion AND SENTIMENT ANALYSIS TECHNiQUES

\begin{tabular}{|l|l|l|}
\hline Author & Feature Selection & Sentiment Analysis Techniques \\
\hline$[9]$ & Not mentioned in paper. & $\begin{array}{l}\text { Used tools (VADER) to identify the percentages of positive, negative, and neutral categories. } \\
\text { They also used Multinomial Naive Bayes and Support Vector machines to determine the polarity of } \\
\text { tweets. }\end{array}$ \\
\hline$[10]$ & Not mentioned in paper. & Used syuzhet as sentiment lexicon to detect sentiments in every sentence in a document. \\
\hline$[11]$ & $\begin{array}{l}\text { Chi-Square, Categorical Proportional } \\
\text { Difference, and Categorical Probability } \\
\text { Proportion Difference }\end{array}$ & $\begin{array}{l}\text { Used Naïve Bayes, Support Vector Machine (SVM), and MaxEnt to classify each labelled paragraph of } \\
\text { Corpus Vi'es into either positive, negative or neutral. }\end{array}$ \\
\hline$[12]$ & $\begin{array}{l}\text { Mentioned, but feature selection was used } \\
\text { in reference to sentiment classification and } \\
\text { extraction. }\end{array}$ & Classified the words into positive, negative or neutral based on the subjectivity of the word. \\
\hline$[17]$ & Not mentioned in paper. & $\begin{array}{l}\text { Used Dictionary-based, Naive Bayes, and SVM algorithms as classifiers in order to assist classification } \\
\text { of test data as positive, negative, and neutral. }\end{array}$ \\
\hline$[18]$ & Not mentioned in paper. & $\begin{array}{l}\text { Used SentiStrength tool and the NRC dictionary for extracting sentiment and emotion based on their } \\
\text { polarities and vectors. }\end{array}$ \\
\hline$[19]$ & Not mentioned in paper. & Used Lexicon and Naïve Bayes algorithm to identify sentiments. \\
\hline$[20]$ & Not mentioned in paper. & Used context-based customized dictionaries. \\
\hline
\end{tabular}

Researchers in [26] have hybridised ant colony optimisation (ACO) and K-nearest neighbour (KNN) as a feature selection technique to produce an optimum feature set that can help to yield high classification and clustering accuracy. They used electrical product data from Nokia, Canon, Apex, Creative, and Nikon. This study used the grammatical structure method which consist the type of reliance and post tagger to identify sentiment word and its relationship with the features contained in the user's review. The combination of ACO-KNN as feature selection has yielded an optimised feature subset and improved classification accuracy.

\section{CONCLUSION}

Based on Table I, most of the reviewed studies did not specify their feature selection method. As for sentiment analysis techniques, several studies have used tools, such as Vader, syuzhet, dictionary-based, and sentiment classification techniques, such as Naïve Bayes, and support vector machines. Based on this literature review, the use of swarm algorithm technique for feature selection is common in different domains, such as product, movie, Twitter, restaurant, and online communication. The results of several experiments in this review have shown that the swarm algorithm technique can produce relevant and high quality feature subsets, which could improve the accuracy of sentiment classification. The results of this review suggest that swarm algorithm technique should be used for feature selection in propaganda domains because this technique has the potential to produce a subset of quality and relevant features. This can help increase the accuracy of sentiment classification. Therefore, more detailed studies and experiments on the use of the swarm algorithm technique for feature selection in propaganda domain need to be conducted to prove that this technique is capable of producing a subset of quality features that are of relevance; thus, increasing the accuracy of the classification of sentiments.

\section{ACKNOWLEDGMENT}

The authors gratefully acknowledge Universiti Pertahanan Nasional Malaysia and the Skim Geran Penyelidikan Jangka Pendek Fasa 2/2018 for supporting this research project through grant no. UPNM/2018/GPJP/2/TK/14.

\section{REFERENCES}

[1] C. R. Wright, Mass communication: A sociological perspective. 1959.

[2] Ron D. (Ed). McLauri, Military propaganda: Psychological warfare and operations. New York: Praeger; First Edition edition, 1982.

[3] R. Di Tella, S. Galiani, and E. Schargrodsky, "Reality versus propaganda in the formation of beliefs about privatization," Journal of Public Economics, 2012.

[4] M. Ginsberg, "Propaganda Art as a Powerful Weapon for Promoting Nationalism, Patriotism and Hatred Towards the Enemy," in Inside the World's Major East Asian Collections: One Belt, One Road, and Beyond, 2017.

[5] LiteraryDevices Editors, "Propaganda," 2014. [Online]. Available: https://literarydevices.net/propaganda/.

[6] E. W. Fellows, “' Propaganda :' History of a Word Author ( s ): Erwin W Fellows Published by: Duke University Press Stable URL: http://www.jstor.org/stable/454039 C HANGES IN THE MEANING attached to a word may be associated with," vol. 34, no. 3, pp. 182-189, 2017

[7] Jowet, "How To Analyze," pp. 269-288, 2009.

[8] A. Abbasi, H. Chen, and A. Salem, "Sentiment Analysis in Multiple Languages: Feature Selection for Opinion Classification in Web forums," ACM Transactions on Information Systems, vol. 26, no. 3, pp. 1-34, 2008.

[9] J. Ramteke, S. Shah, D. Godhia, and A. Shaikh, "Election result prediction using Twitter sentiment analysis," in Proceedings of the International Conference on Inventive Computation Technologies, ICICT 2016, 2017.

[10] D. Liu and L. Lei, "The appeal to political sentiment: An analysis of Donald Trump's and Hillary Clinton's speech themes and discourse strategies in the 2016 US presidential election," Discourse, Context and Media, 2018.

[11] Caio Magno Aguiar Carvalho, Hitoshi Nagano, and Allan Kardec Barros, "A Comparative Study for Sentiment Analysis on Election Brazilian News," in Proceedings of the 11th Brazilian Symposium in Information and Human Language Technology, 2017, pp. 103-111. 
[12] M. K. Akhmedov Khumoyun, Y. Cui, and H. Lee, "Storm based Realtime Analytics Service on Propaganda and Sentiment Analysis of Political Tweets on Cloud Computing Environment," in International Conference on Engineering Technologies and Big Data Analytics, 2016, pp. $61-65$.

[13] S. R. Ahmad, A. A. Bakar, and M. R. Yaakub, "A review of feature selection techniques in sentiment analysis," Intelligent Data Analysis, vol. 23, no. 1, pp. 159-189, 2019.

[14] B. Liu, Sentiment Analysis and Opinion Mining. Morgan \& Claypool Publishers, 2012.

[15] M. Jockers, "syuzhet: Extracts Sentiment and Sentiment-Derived Plot Arcs from Text," The R-Project, 2016.

[16] M. E. Roberts et al., "Structural Topic Models for Open-Ended Survey Responses," American Journal of Political Science, 2014.

[17] P. Sharma and T. S. Moh, "Prediction of Indian election using sentiment analysis on Hindi Twitter," in Proceedings - 2016 IEEE International Conference on Big Data, Big Data 2016, 2016.

[18] E. Kušen and M. Strembeck, "Politics, sentiments, and misinformation: An analysis of the Twitter discussion on the 2016 Austrian Presidential Elections," Online Social Networks and Media, 2018.

[19] B. Joyce and J. Deng, "Sentiment analysis of tweets for the 2016 US presidential election," in 2017 IEEE MIT Undergraduate Research Technology Conference, URTC 2017, 2018.
[20] S. Hari, D. Kolagani, and M. B. A. Student, "Identifying Trending Sentiments in the 2016 U . S . Presidentialelection: A Case Study Of Twitter Analytic," Issues in Information Systems, vol. 18, no. 2, pp. 80 86, 2017.

[21] A. Kumar, R. Khorwal, and S. Chaudhary, "A Survey on Sentiment Analysis using Swarm Intelligence," vol. 9, no. October, 2016.

[22] L. Shang, Z. Zhou, and X. Liu, "Particle swarm optimization-based feature selection in sentiment classification," Soft Computing, vol. 20, no. 10, pp. 3821-3834, 2016.

[23] L. Goel and A. Prakash, "Sentiment Analysis of Online Communities Using Swarm Intelligence Algorithms," in Proceedings - 2016 8th International Conference on Computational Intelligence and Communication Networks, CICN 2016, 2017.

[24] M. H. Aghdam and S. Heidari, "Feature Selection Using Particle Swarm Optimization in Text Categorization," Journal of Artificial Intelligence and Soft Computing Research, vol. 5, no. 4, pp. 231-238, 2015.

[25] J. P. Nirmala Devi K, "Sentiment Classification Using SVM And PSO," International Journal of Advanced Engineering Technology E- Int J Adv Engg Tech VII/Issue II, pp. 411-413, 2016.

[26] S. R. Ahmad, A. A. Bakar, and M. R. Yaakub, "Ant colony optimization for text feature selection in sentiment analysis," Intelligent Data Analysis, vol. 23, no. 1, pp. 133-158, 2019. 